\title{
Real-world use and acceptance of rituximab biosimilars in non-Hodgkin lymphoma in an oncologist network in Germany
}

\author{
Burkhard Otremba*,1, Jens Borchardt² ${ }^{2}$ Andra Kuske ${ }^{3}$, Maike Hollnagel-Schmitz ${ }^{3}$ \& Florian \\ O Losch ${ }^{3}$ \\ ${ }^{1}$ Onkologische Praxis Oldenburg/Delmenhorst, Oldenburg, Germany \\ ${ }^{2}$ Onkotrakt AG, Hamburg, Germany \\ ${ }^{3}$ Medical Oncology, Hexal AG, Holzkirchen, Germany \\ *Author for correspondence: Tel.: +49 441770598 0; Fax: +49 4417705 98 10; otremba@onkopraxis-oldenburg.de
}

\begin{abstract}
Aim: Present real-world data for rituximab (biosimilar and reference)-containing regimens in extrapolated indications in non-Hodgkin lymphoma (NHL)/chronic lymphocytic leukemia (CLL). Patients \& methods: Data collected from office-based oncologic practices in Germany (July 2017-June 2019). Results: Of 1741 patients, 1241 had NHL; 500 had CLL. Of 7595 therapy cycles, $28.3 \%$ used reference rituximab; 55.2\% used rituximab biosimilars; $2.0 \%$ used subcutaneous rituximab; $14.5 \%$ used rituximab, not otherwise specified. Rituximab biosimilars were used across all indications; $57.3 \%$ of cycles were administered in extrapolated indications. Over 24 months, the proportion of rituximab prescriptions that were for biosimilars increased from 12.0 to $83.0 \%$. Conclusion: Our real-world data in NHL and CLL depicts increasing use of rituximab biosimilars across multiple treatment protocols, including extrapolated indications.
\end{abstract}

First draft submitted: 2 March 2020; Accepted for publication: 25 March 2020; Published online: 14 April 2020

Keywords: biosimilar • chronic lymphocytic leukemia • non-Hodgkin lymphoma • real-world • rituximab

Rituximab was the first monoclonal antibody to be approved for therapeutic use in oncology and remains a fundamental component of the current treatment paradigm for non-Hodgkin lymphoma (NHL) [1]. In chronic lymphocytic leukemia (CLL), although recent developments have seen treatment become dominated by small molecule kinase inhibitors (e.g., ibrutinib, venetoclax), rituximab in combination with these and other agents remains a useful option [2].

The first rituximab biosimilars (Rixathon ${ }^{\circledR}$ [Sandoz rituximab], developed by Sandoz, Holzkirchen, Germany; and Truxima ${ }^{\circledR}$ [Celltrion rituximab], developed by Celltrion, Incheon Metropolitan City, South Korea) were approved by the EMA in 2017. Approval was based on the totality of evidence for biosimilarity derived from a comprehensive comparability exercise with the reference medicine (MabThera ${ }^{\circledR} /$ Rituxan ${ }^{\circledR}$ [reference rituximab], Roche, Basel, Switzerland/Genentech, CA, USA) [3-6]. This comparability exercise included extensive physicochemical and structural evaluations, as well as data from preclinical and clinical pharmacokinetic $(\mathrm{PK})$, pharmacodynamic and immunogenicity assessments.

For both biosimilars, the final step in the development process was a confirmatory Phase III clinical trial in patients with advanced follicular lymphoma (FL) [7-13]. In the Phase III ASSIST-FL trial, 627 patients were randomized to receive either Sandoz rituximab or EU-sourced reference rituximab [9]. For Celltrion rituximab, the pivotal trial (CT-P10 3.3) featured 140 patients, who were randomized to receive either the biosimilar or US-sourced reference rituximab [13]. In both trials, rituximab was administered in combination with cyclophosphamide, vincristine, prednisolone chemotherapy. Trials in rheumatoid arthritis have also been conducted for both biosimilars. For Celltrion rituximab, one Phase I trial, including a long-term safety and efficacy extension, and one Phase III trial were conducted, including a total of 584 patients [14-17]. For Sandoz rituximab, a Phase II PK equivalence study and a Phase III switching study to establish safety and immunogenicity were conducted, with a total of 419 patients included across the pair $[11,18]$. These clinical trials were conducted in carefully selected indications that were likely

Future Medicine 
to be the most sensitive to detect differences between the biosimilar and reference medicine. Demonstration of similarity in these trials, coupled with extensive evidence of similarity from other tests, allowed the extrapolation of data with the reference medicine to the biosimilar in other nontested indications [19]. This aspect of biosimilar development, endorsed by health authorities worldwide, reduces the requirement for clinical testing, allowing biosimilars to be produced more affordably than reference medicines.

Development and introduction of biosimilars that are similar in structure, function, immunogenicity, efficacy and safety to the reference biological agent, but available at a lower list price, has the potential to provide savings for healthcare systems, to broaden patient access to biological therapies, and to support the sustainability of cancer care [20-23]. In Germany, biosimilars are available at a substantially lower cost than the reference medicine (as of March 2020, Sandoz rituximab is available at a saving of $11 \%$ vs the reference medicine).

While clinical trial data with rituximab biosimilars are available in FL and rheumatoid arthritis, by definition no data are available for extrapolated indications [9,12,13,24]. Since its first launch in 1998, extensive real-world experience with reference rituximab has been reported [25-29]. Real-world evidence is collected during routine clinical practice, outside the tightly controlled environment of a clinical trial and, therefore, helps to fill the knowledge gap between clinical trial experience and routine clinical practice. This can help to address the fact that patient populations may differ significantly between clinical trials and in the real world in terms of demographics and disease characteristics, including age, gender, ethnicity, disease severity, comorbidity burden, concomitant medication and treatment compliance [30]. Payers, physicians and other stakeholders may also use real-world evidence of effectiveness and health outcomes data to inform prescribing and reimbursement decisions [31], and clinical trials increasingly include economic end points and/or analyses to evaluate cost-effectiveness.

Here we present data on current real-world treatment patterns with rituximab biosimilars and reference rituximab in patients with NHL and CLL in Germany.

\section{Patients \& methods}

Patient demographics and prescription data for rituximab-based regimens in patients with NHL and CLL were collected electronically, and analyzed as part of the multicenter software-based epidemiological healthcare research project 'LiveTicker' of Onkotrakt AG, which has been in existence since 2011. In this system, defined patient and treatment data from routine care are prospectively documented using 'Oncotrace' software, a package for automated documentation and data evaluation. Since therapy planning is the central element of the documentation, these consecutively collected data are less prone to failure in relation to a possible selection bias compared with a retrospective case study and parallel documentation. The uniform electronic data acquisition thus enables representative and promptly available analyses of the real supply situation.

Data collection began on 1 July 2017 and is ongoing. For this report, data were captured up to the end of June 2019. In total, 71 physicians across 23 centers participated in data collection. Investigators were office-based oncologists practicing in Germany. Treatment data were anonymized by the participating centers prior to entry into the database. WHO International Classification of Diseases codes were used to group patients by cancer type (Supplementary Table 1). Analyses generally compare data from patients treated with reference rituximab, a rituximab biosimilar ('Sandoz rituximab' or 'Celltrion rituximab') and nonspecified rituximab antibodies (rituximab $\mathrm{n} / \mathrm{s}$ ), which are all intravenously administered. Patients treated with the subcutaneous (SC) formulation of reference rituximab (reference rituximab SC) were included in this analysis but comprise less than $2 \%$ of the population. A preliminary longitudinal analysis was conducted by comparing rituximab use in the first (July 2017) and last (June 2019) months of the available dataset. All data were analyzed descriptively using SPSS software. Institutional review board approval for the study was obtained from the ethics committee of the ÄK Nordrhein, and the study was also endorsed by ethics committees of the individual study centers in Baden-Wuerttemberg, Brandenburg, Hamburg, Mecklenburg-West Pomerania, Lower Saxony, Rhineland-Palatinate, Saxony, Schleswig-Holstein, Thuringia and Westphalia Lippe.

\section{Results}

In total, 1741 patients were included in the database; 1241 patients had NHL and 500 patients had CLL. International Classification of Diseases codes confirmed that among patients with NHL, 323 (18.6\%) had FL, 243 (14.0\%) had diffuse large B-cell lymphoma (DLBCL) and 675 (38.8\%) had other types of (or codes for) NHL, which included 102 patients (5.9\%) with marginal zone lymphoma, 63 (3.6\%) with mantle cell lymphoma, 47 (2.7\%) with 'other nonfollicular lymphoma', 25 (1.4\%) with natural killer/T (NK/T) cell lymphoma and 438 


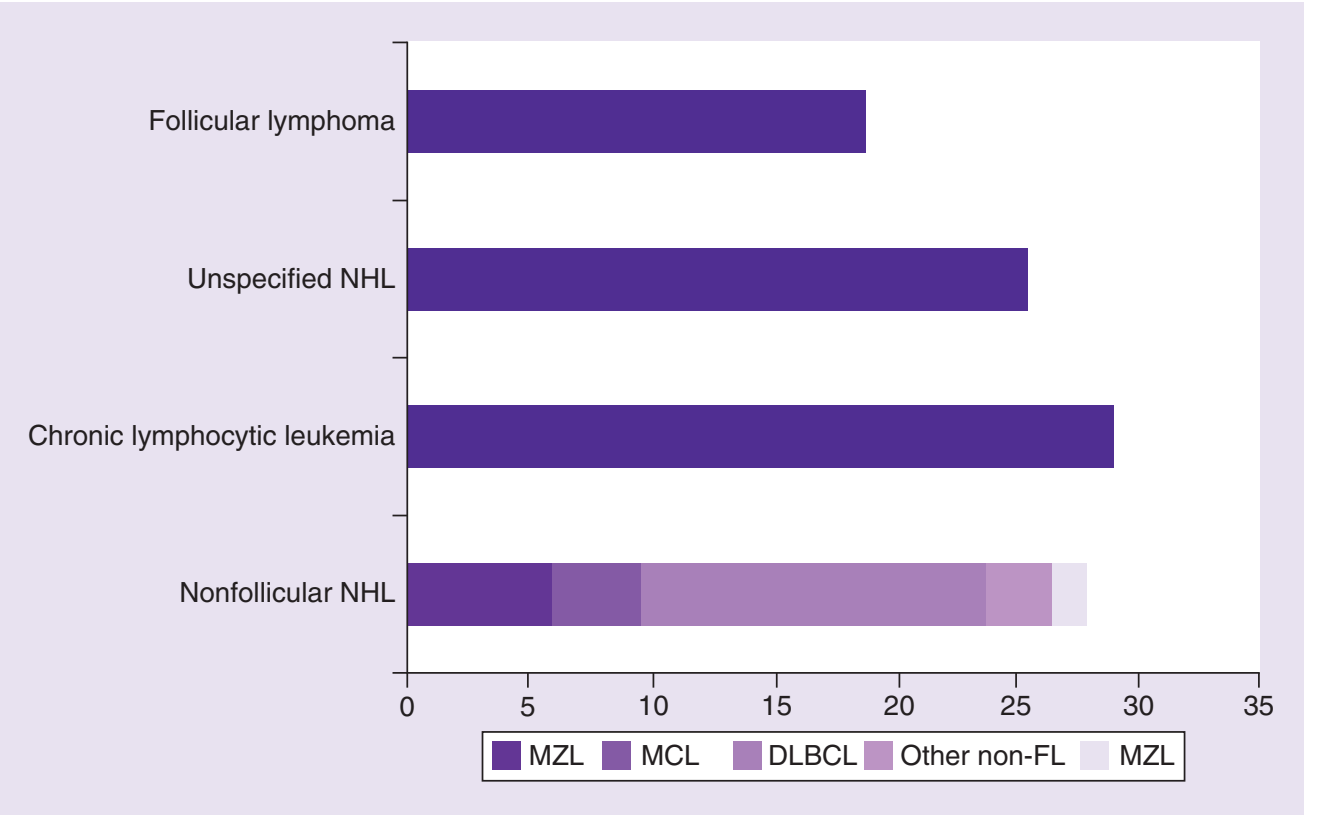

Figure 1. Distribution of patients included in the database according to International Classification of Diseases code indication.

DLBCL: Diffuse large B-cell lymphoma; FL: Follicular lymphoma; MCL: Mantle cell lymphoma; MZL: Marginal zone lymphoma; NHL: Non-Hodgkin lymphoma.

\section{Table 1. Patient demographics.}

\begin{tabular}{|c|c|c|c|}
\hline Characteristics & All patients $(n=1741)$ & Patients with NHL $(n=1241)$ & Patients with CLL $(n=500)$ \\
\hline \multicolumn{4}{|l|}{ Age (years) } \\
\hline $11-20$ & $5(0.3)$ & $5(0.4)$ & $0(0.0)$ \\
\hline $21-30$ & $11(0.6)$ & $11(0.9)$ & $0(0.0)$ \\
\hline $31-40$ & $19(1.1)$ & $18(1.5)$ & $1(0.2)$ \\
\hline $41-50$ & $93(5.3)$ & $80(6.4)$ & $13(2.6)$ \\
\hline $51-60$ & $277(15.9)$ & $209(16.8)$ & $68(13.6)$ \\
\hline $61-70$ & $387(22.2)$ & $279(22.5)$ & $107(21.4)$ \\
\hline $71-80$ & $632(36.3)$ & $424(34.2)$ & $207(41.4)$ \\
\hline $81-90$ & $307(17.6)$ & $208(16.8)$ & $101(20.2)$ \\
\hline$\geq 91$ & $10(0.6)$ & $7(0.6)$ & $3(0.6)$ \\
\hline \multicolumn{4}{|l|}{ Sex } \\
\hline Male & $992(57.0)$ & $692(55.8)$ & $300(60.0)$ \\
\hline Female & $749(43.0)$ & $549(44.2)$ & $200(40.0)$ \\
\hline
\end{tabular}

Data represented as $n(\%)$.

CLL: Chronic lymphocytic leukemia; NHL: Non-Hodgkin lymphoma.

(25.2\%) with 'unspecified NHL' (Figure 1). Most patients (58.8\%) were aged 60-80 years, $57.0 \%$ of patients were male, and $43 \%$ were female (Table 1 ).

A total of 38 different rituximab-containing therapeutic protocols were used, including bendamustine plus rituximab (BR) - 426 patients; rituximab plus cyclophosphamide, doxorubicin, vincristine and prednisolone (R-CHOP) - 337 patients; rituximab maintenance therapy - 307 patients; rituximab monotherapy 223 patients; $\mathrm{BR}+$ methotrexate -43 patients; fludarabine plus cyclophosphamide and rituximab - 38 patients; $\mathrm{R}-\mathrm{CHOP}+$ methotrexate -27 patients; rituximab plus methotrexate -23 patients; rituximab plus cyclophosphamide, doxorubicin, vincristine, etoposide and prednisolone (R-CHOEP) - 22 patients; rituximab plus chlorambucil - 20 patients; and 'Other' regimens - 74 patients (Figure 2). The 'Other' data series comprises all other rituximab-containing protocols $(\mathrm{n}<20$, range $1-12$; Table 2$)$. In addition, 15 protocols used other anti-CD20 


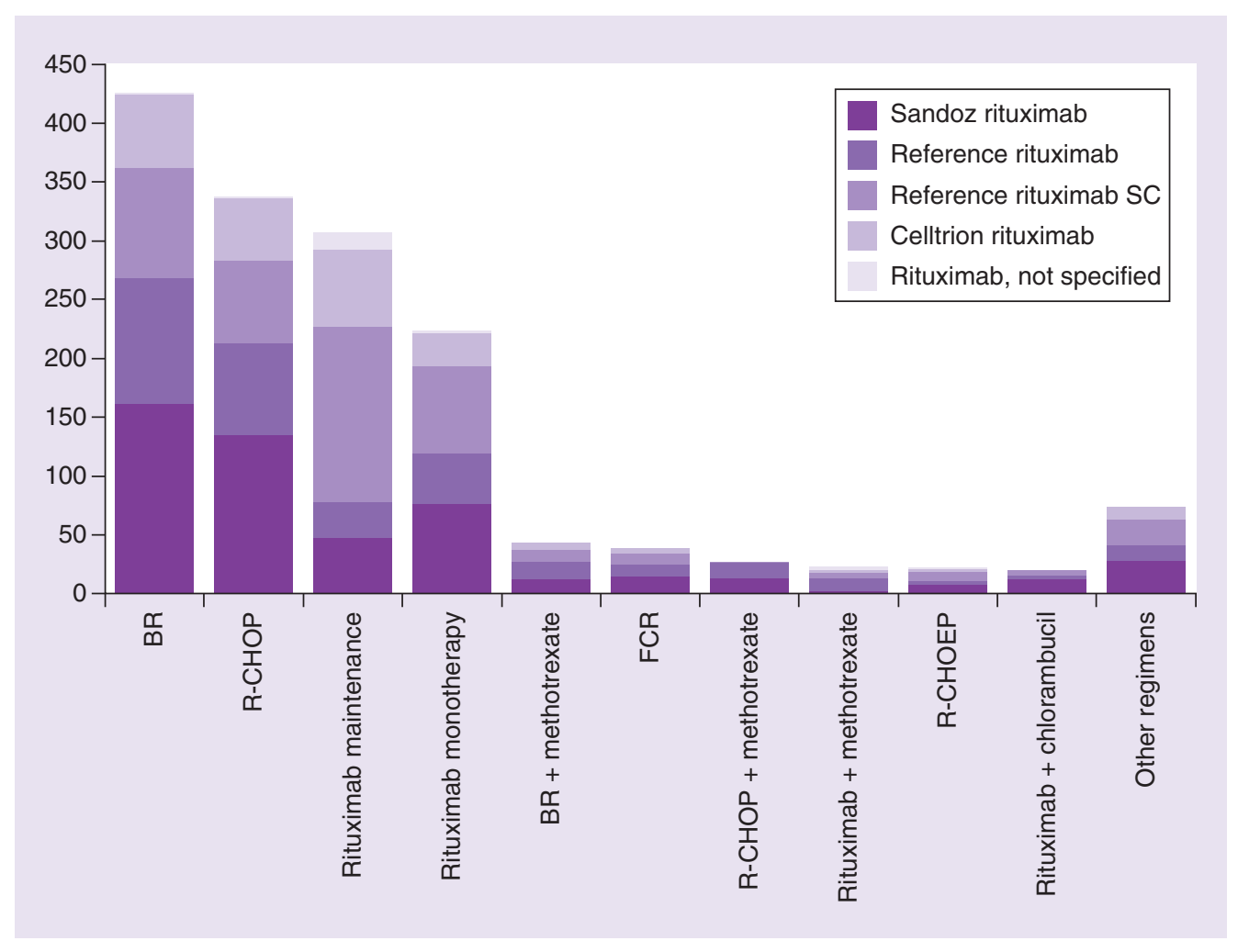

Figure 2. Rituximab use by product and therapeutic protocol.

BR: Bendamustine plus rituximab; FCR: Fludarabine plus cyclophosphamide and rituximab; n/s: Not specified; R-CHOP: Rituximab plus cyclophosphamide, doxorubicin, vincristine and prednisolone; SC: Subcutaneous.

\section{Table 2. Most frequent rituximab protocols identified in the Onkotrakt database.}

Treatment protocol Patients (n)

\section{Most frequent ( $>20$ patients)}

Bendamustine + rituximab (BR)

426

Rituximab + cyclophosphamide + doxorubicin + vincristine + prednisolone (R-CHOP) 426

Rituximab maintenance 337

Rituximab monotherapy 307

$\mathrm{BR}+$ methotrexate 223

Fludarabine + cyclophosphamide + rituximab

R-CHOP + methotrexate

Methotrexate + rituximab

Rituximab + cyclophosphamide + doxorubicin + vincristine + etoposide + prednisolone

antibodies such as obinutuzumab (179 patients) and ofatumumab (17 patients).

Of 7595 total rituximab cycles in all patients, a rituximab biosimilar was used in $55.2 \%$ of cycles $(31.8 \%$ Sandoz rituximab, $23.4 \%$ Celltrion rituximab), reference rituximab in $28.3 \%$ of cycles and rituximab $\mathrm{n} / \mathrm{s}$ in $14.5 \%$ of cycles. In patients with FL, a rituximab biosimilar was used in $48.6 \%$ of cycles $(28.1 \%$ Sandoz rituximab, $20.5 \%$ Celltrion rituximab) and in patients with DLBCL, a rituximab biosimilar was used in $70.0 \%$ of cycles (45.0\% Sandoz rituximab, 25.1\% Celltrion rituximab). In the total population, only $154(2.0 \%)$ cycles of reference rituximab $\mathrm{SC}$ were administered across the same period.

A rituximab biosimilar was used in $42.4 \%$ of patients with FL (25.5\% Sandoz rituximab, 16.9\% Celltrion rituximab), and in $64.3 \%$ of patients with DLBCL (42.1\% Sandoz rituximab, 22.2\% Celltrion rituximab; Figure 3A). 


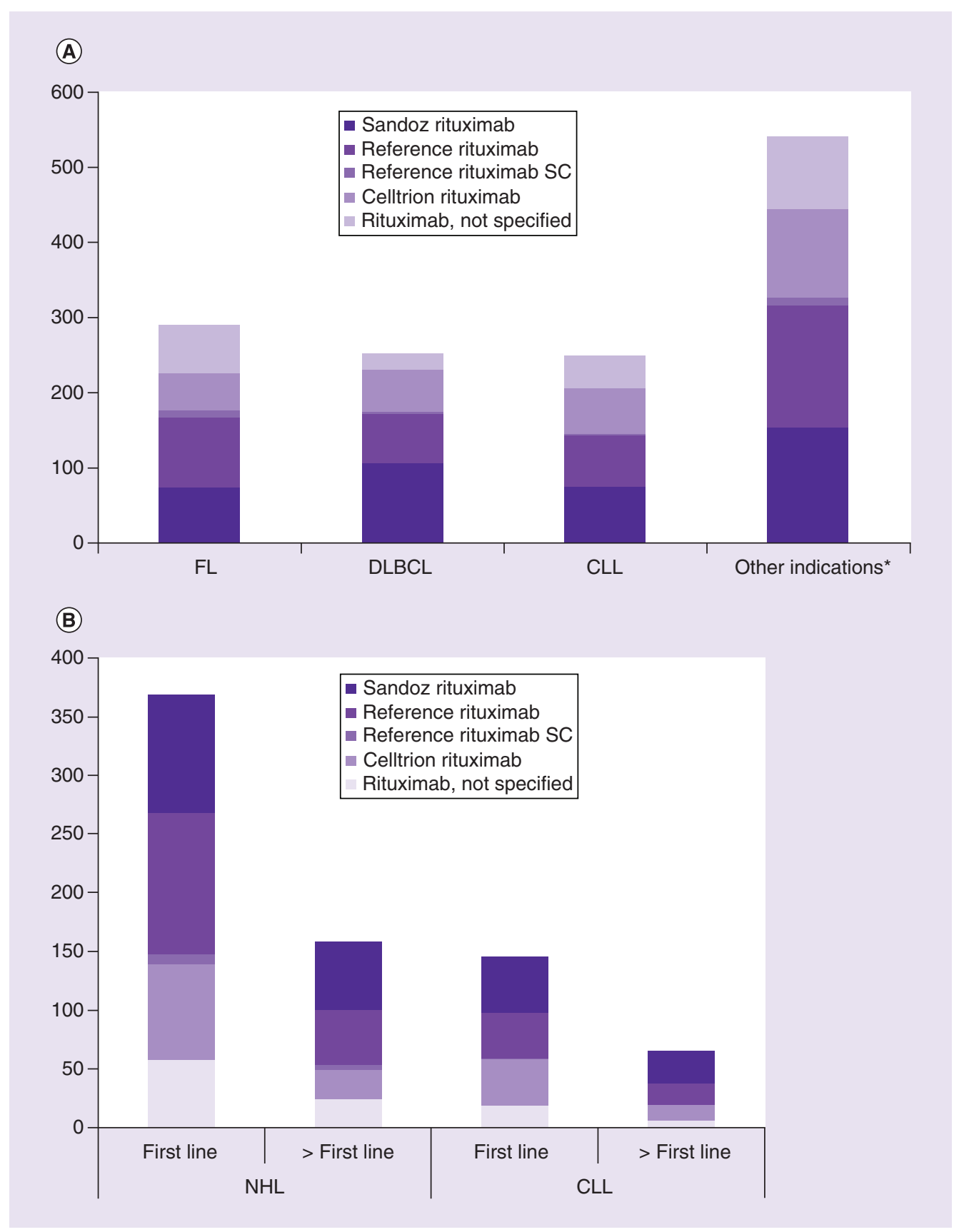

Figure 3. Rituximab use. (A) indication and (B) line of therapy.

*Marginal zone lymphoma, mantle cell lymphoma, 'other nonfollicular lymphoma', NK/T cell lymphoma, 'unspecified NHL'.

CLL: Chronic lymphocytic leukemia; DLBCL: Diffuse large B-cell lymphoma; FL: Follicular lymphoma; NHL: Non-Hodgkin lymphoma; NK/T cell: Natural killer/T cell; SC: Subcutaneous.

In total, $54.6 \%$ of patients with extrapolated indications received a rituximab biosimilar; a rituximab biosimilar was used in $50.0 \%$ of patients in the 'others' group ( $28.4 \%$ Sandoz rituximab, $21.6 \%$ Celltrion rituximab) and $54.8 \%$ of patients in the CLL group (28.4\% Sandoz rituximab, 21.6\% Celltrion rituximab; Figure 3A). For Sandoz rituximab and Celltrion rituximab, an increase in the number of cycles administered was observed between 2017 and 2019, while for reference rituximab, a corresponding decrease in the number of cycles administered was observed over this time period (Figure 4). The increases in cycles administered were most pronounced for Sandoz rituximab. These changes were consistently observed across all disease-related subgroups. 


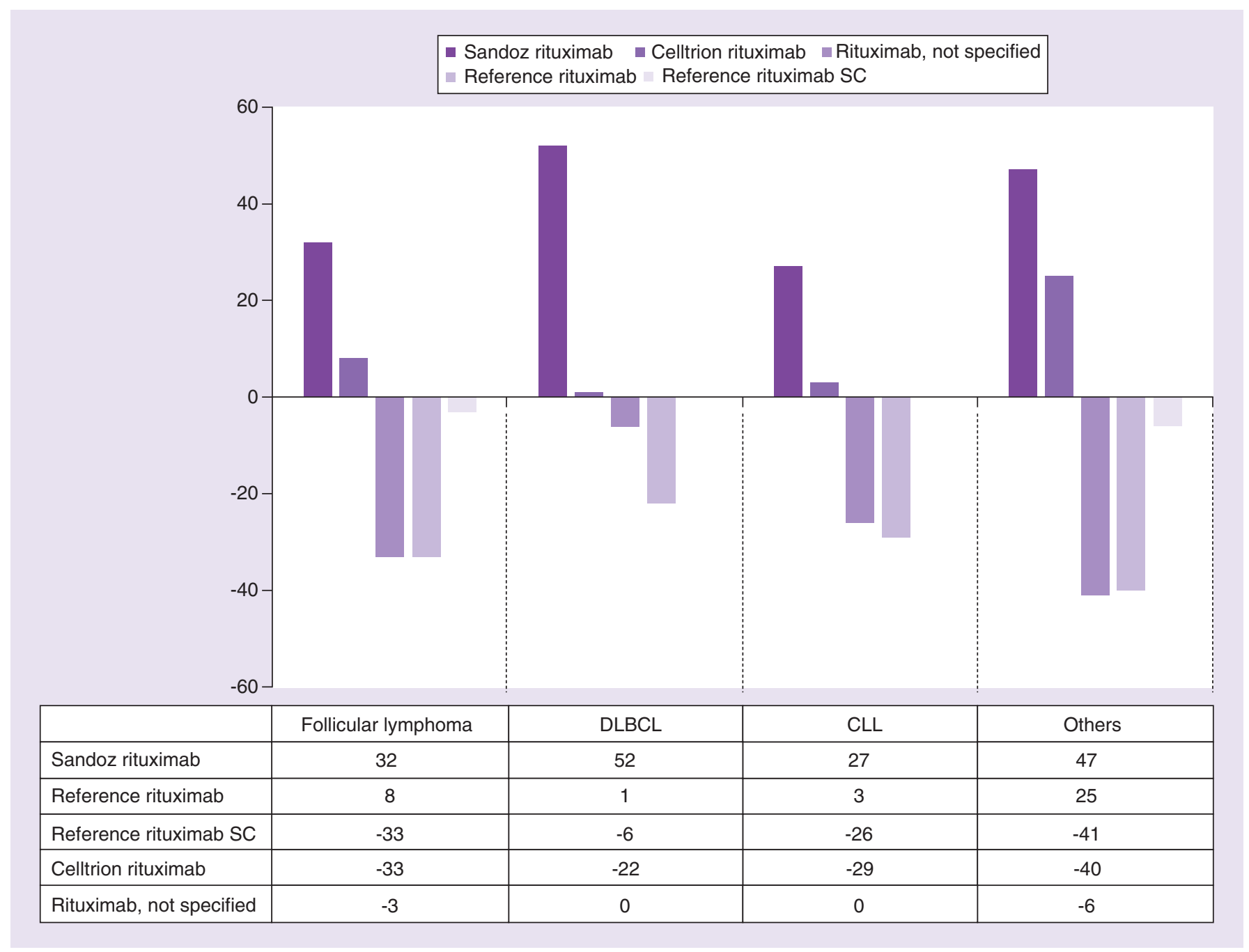

Figure 4. Differences in number of cycles administered for each rituximab formulation between 2017 and 2019. CLL: Chronic lymphocytic leukemia; DLBCL: Diffuse large B-cell lymphoma; SC: Subcutaneous.

In NHL, a rituximab biosimilar was used in $49.2 \%$ of patients for first-line treatment ( $27.2 \%$ Sandoz rituximab, $22 \%$ Celltrion rituximab) and in $52.5 \%$ of patients receiving second-line or later treatment $(36.7 \%$ Sandoz rituximab, 15.8\% Celltrion rituximab; Figure 3B). In CLL, a rituximab biosimilar was used in $59.3 \%$ of patients for first-line treatment (32.4\% Sandoz rituximab, 26.9\% Celltrion rituximab) and $61.5 \%$ of patients for secondline or later treatment (41.5\% Sandoz rituximab, 20.0\% Celltrion rituximab; Figure 3B). Overall, in patients with NHL or CLL, a rituximab biosimilar was used in 52\% at first line and $55 \%$ at second line or later.

Analysis of patients switching from one rituximab medicine to another indicates that switches can occur from reference medicine to biosimilar ( $\mathrm{n}=31,47.0 \%)$, between different biosimilar medicines $(\mathrm{n}=14,21.2 \%)$, and from biosimilar to reference medicine ( $\mathrm{n}=18,27.3 \%$; Figure 5). In total, 316 switches were recorded in the database; 193 were within therapy and 123 were to a new therapy.

Within the observation period, the proportion of rituximab biosimilar prescriptions in the total population increased from $12.0 \%$ (July 2017) to $83.0 \%$ (June 2019), comprising an increase in prescriptions of Sandoz rituximab from 1.2 to $57.4 \%$ and an increase in prescriptions of Celltrion rituximab from 10.7 to $25.5 \%$. Across the same period, the proportion of reference medicine prescriptions decreased from 51.5 to $15.6 \%$, and rituximab $\mathrm{n} / \mathrm{s}$ prescriptions from 33.4 to $1.1 \%$ (Figure $6 \mathrm{~A}$ ). These prescription patterns were generally similar in patients with DLBCL, CLL, unspecified non-FL, marginal zone lymphoma, mantle cell lymphoma and NK/T cell lymphoma (Figure 6B-D). 


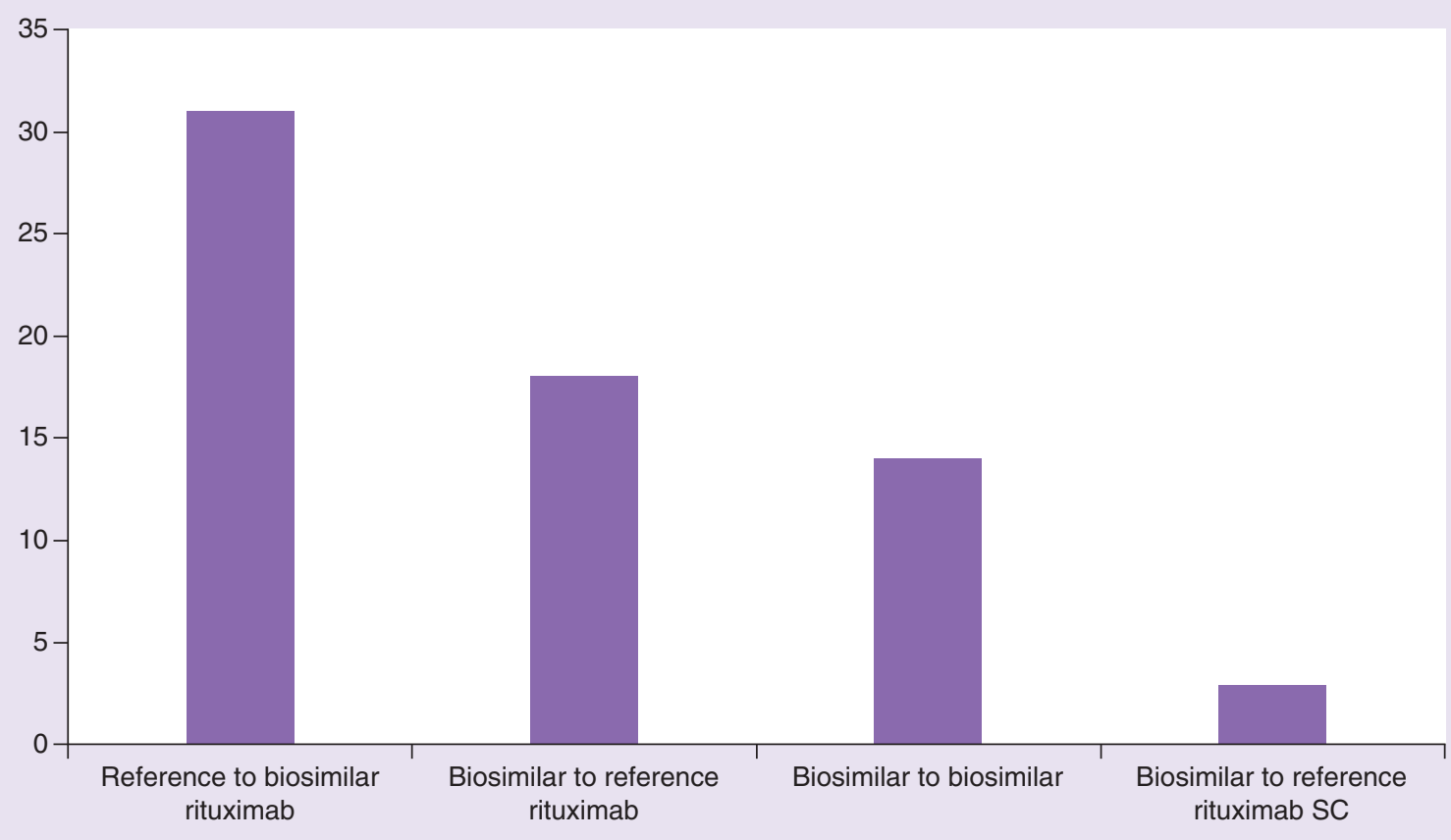

Figure 5. Switching data between reference and biosimilar or between biosimilar rituximab medicines.

SC: Subcutaneous.

\section{Discussion}

This study describes the evolution of the use of biosimilar rituximab in Germany in the first 24 months after approval. Our data show that approximately 38 different rituximab-containing therapeutic protocols were used 1540 times to treat patients with NHL and CLL by physicians across Germany, compared with 15 protocols for obinutuzumab and ofatumumab, which were used 179 times. Rituximab biosimilars are increasingly being used for the treatment of patients with NHL and CLL, indicating a high acceptance of biosimilars, even in extrapolated indications. Overall, biosimilars were used in $>50 \%$ of patients both at first line and at second line or greater. From July 2017 to June 2019, the proportion of biosimilar rituximab prescriptions increased sevenfold, primarily driven by an increase in prescriptions of Sandoz rituximab. This suggests that, with increased familiarity over time, the prescribing physicians in this network became less conservative about biosimilar use. This observation also held in curative settings and for less common rituximab protocols. Overall, however, the data demonstrate the considerable heterogeneity of the treatment landscape, with a wide range of different patient types, which requires an individualized treatment approach.

It is notable that rituximab biosimilars seemed to be used more frequently in the curative setting of DLBCL ( $70.0 \%$ of cycles) than in FL, a more indolent lymphoma ( $48.6 \%$ of cycles). In fact, rituximab biosimilars were the most commonly administered rituximab products in DLBCL, being used to treat nearly $65 \%$ of patients across the full study period, with $90 \%$ of patients receiving a rituximab biosimilar by the end of the observation time. This pattern may be an artefact of the 'snapshot' study design, however. We observed that switches from reference medicine to biosimilar were rare, being seen in only 31 patients. Therefore, in the majority of cases, biosimilars were initiated in new patients entering treatment for the first time. Because FL is an indolent lymphoma, fewer new cases might be observed than with the more aggressive lymphoma DLBCL. Therefore, more patients with DLBCL were initiated on therapy during the study, with a correspondingly greater chance of being initiated on a rituximab biosimilar. Use of rituximab was also common in patients with CLL, highlighting the continuing perceived usefulness of anti-CD20 targeting therapy in the era of small molecule kinase inhibitors such as ibrutinib, idelalisib and venetoclax.

The reason for prescribing a biosimilar as opposed to the reference medicine was not recorded in our study, although we surmise that the increases in prescriptions noted over the course of the study were motivated mostly by the prospect of cost savings, as well as the novelty of biosimilars, with both biosimilars approved for use in Europe 

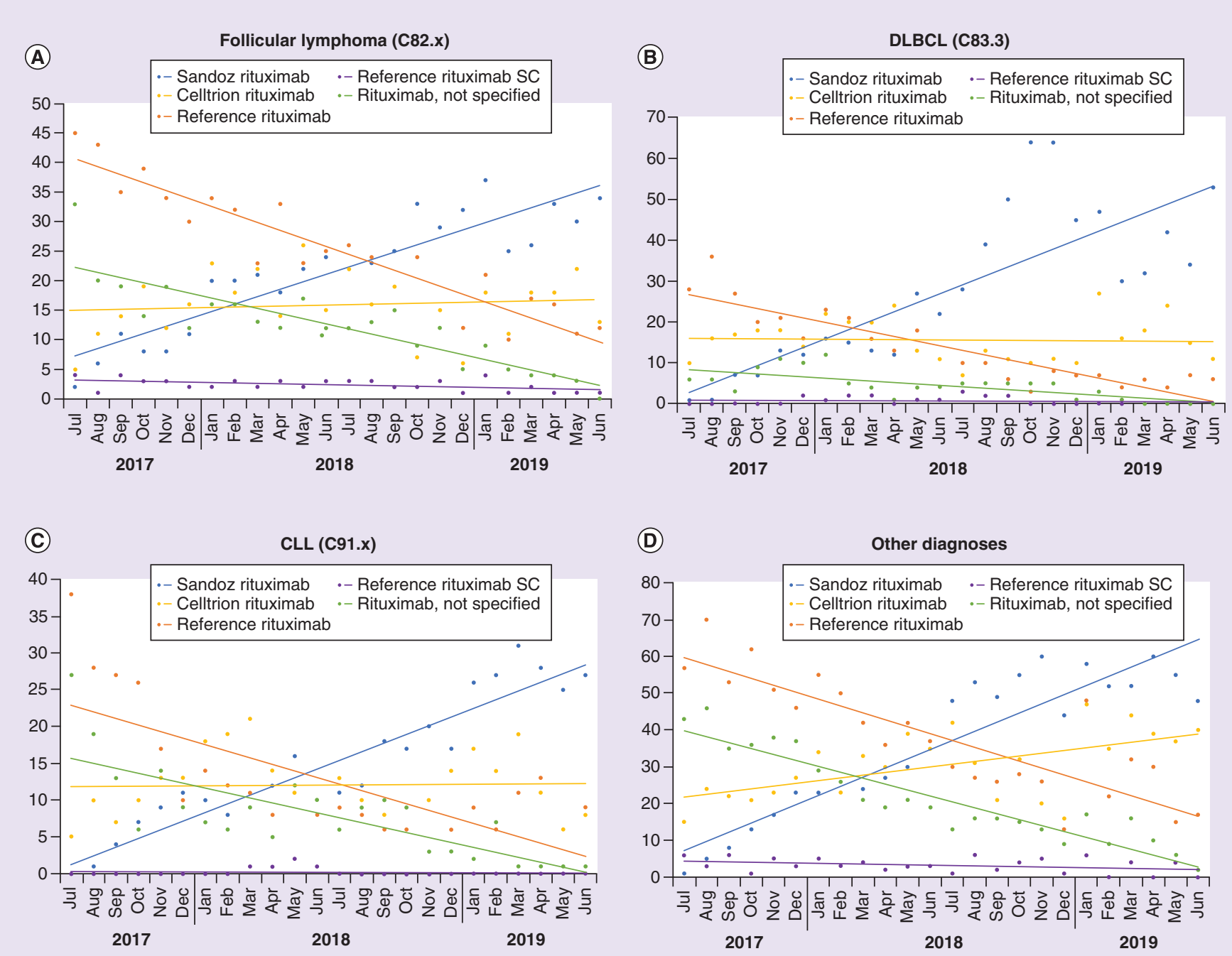

Figure 6. Time course of rituximab prescriptions. (A) Follicular lymphoma, (B) DLBCL, (C) CLL, (D) Other (unspecified non-Follicular lymphoma, Marginal zone lymphoma, Mantle cell lymphoma, Natural killer/T cell cell lymphoma). Data presented as polynomial trend lines of the percentage prescriptions per month.

CLL: Chronic lymphocytic leukemia; DLBCL: Diffuse large B-cell lymphoma; NHL; SC: Subcutaneous.

at around the time of study start. In a prior study, it was shown that, from a fixed list of choices, the top three reasons for prescribing biosimilar rituximab in patients with NHL were 'approved standard of care' (71\%), 'proven efficacy' (50\%) and 'on formulary/hospital policy' (32\%) [32].

In our study, there seemed to be a tendency for greater use of Sandoz rituximab compared with Celltrion rituximab. From our data, it is not possible to explain this phenomenon; certainly, it was not driven by the list price, and there are few if any other differentiating factors between the two medicines. Overall, use of biosimilars in preference to the reference medicine may have been motivated by the prospect of cost savings; for Sandoz rituximab, the reduction in list price compared with the reference medicine is 11\% (March 2020 data). Given the high cost of rituximab overall, this represents a significant saving that can potentially be used for alternative therapies or reinvested in other aspects of healthcare.

The safety of switching between biosimilar and reference medicines has been a matter of debate in recent years. However, current EMA recommendations state that there is no clinical evidence to suggest any associated risk or a reduction in efficacy [33,34]. In a systematic literature review of outcomes following a switch from biosimilars to reference medicines ( $\mathrm{n}=14,225$ patients), almost all of the 90 included studies reported no differences in immunogenicity, safety or efficacy [35]. Only a small number of switches were recorded in our study, suggesting 
that physicians generally maintain their original treatment choice. The switches that did occur included the entire gamut of possible changes, including reference medicine to biosimilar, between different biosimilar medicines, and from biosimilar to reference medicine. The reason for switching was not recorded in our study, and owing to the small number of switches, data would not be conclusive.

Biosimilars have the potential to provide significant cost savings for healthcare systems, broaden patient access to biological therapies and support the sustainability of cancer care. The affordability crisis in oncology is believed to be unsustainable, and a greater focus on value, alongside efficacy and safety, needs to be implemented across both healthcare systems and industry to prevent restricted access to life-saving treatments $[36,37]$. Biosimilar and generic medicines provide one option to combat the increasing costs of cancer drugs. However, any improvements in sustainability afforded by biosimilars rely entirely on their uptake [38]. Our results suggest that, after some initial reluctance, the uptake of rituximab biosimilars appears to be increasing, likely fueled by a greater acceptance of biosimilars within the oncology community combined with the economic benefits. However, more time is required to fully realize the benefits of biosimilar drugs in terms of savings and patient access, and to determine whether these savings can be reinvested into the development of innovative therapies. One US cost-saving study in patients with NHL suggests that using reference rituximab SC instead of reference IV rituximab within the R-CHOP regimen elicits both time and cost savings. However, use of biosimilar IV rituximab saved even further on costs versus reference rituximab SC in small- and average-sized patients irrespective of the level of biosimilar discount, and in large patients if discounted by approximately $25 \%$ [39]. An additional study of the economic impact of biosimilars for both rituximab and trastuzumab found that switching to biosimilar rituximab in advanced FL provided a considerable monthly saving (approximately €274), suggesting that rituximab biosimilars are costeffective treatments in this setting [40].

The strength of the current analysis is that in a relatively short time, real-world data regarding treatment protocols were collected using the Onkotrakt network of office-based oncologists. However, the present dataset does not include efficacy or safety data, which could potentially be evaluated in future. Such data would be interesting given that many of the indications treated as part of this study were extrapolated indications for which data were not collected as part of the regulatory approval process for the included biosimilars. The retrospective nature of the study is an important limitation and should be considered when interpreting the data. Retrospective studies such as this use data collected during day-to-day clinical practice, for example, patient medical charts. The data therefore represent a convenient sample, obtained from centers and physicians that are willing to participate, which may not be representative of the entire patient population. It should also be noted that German law requires prescribers to use brand names when prescribing rituximab, so the generic description 'rituximab' should not be present in the dataset. The use of the generic description is expected to decline in future, a trend that we have observed in our data.

In conclusion, this study showed that approximately 38 different rituximab-containing therapeutic protocols are being used in clinics across Germany. Over the last 2 years, biosimilar rituximab has been increasingly used for the treatment of patients with NHL and CLL, suggesting an increasing acceptance of both biosimilars and extrapolation across indications within the oncology community. Additional real-world studies are required to evaluate the longterm effect of biosimilar rituximab on health economics, patient access and the overall sustainability of cancer care.

Supplementary data

To view the supplementary data that accompany this paper please visit the journal website at: www.futuremedicine.com/doi/suppl/10.2217/fon-2020-0180.

\section{Acknowledgments}

The authors thank all participating doctors in the Onkotrakt network and their patients. Statistical support was provided by Ralf Reichelt of Onkotrakt AG. 
involvement with any organization or entity with a financial interest in or financial conflict with the subject matter or materials discussed in the manuscript apart from those disclosed.

Medical writing support, under the direction of the authors, was provided by B Caldwell and P Scutt of Spirit, a division of Spirit Medical Communications Group Limited, Manchester, UK, and funded by Hexal AG.

Ethical conduct of research

Data were collected within the Onkotrakt live-ticker registry, which has approval from the ethics committee of the Ärztekammer Nordrhein (approval number 2017259).

Open access

This work is licensed under the Attribution-NonCommercial-NoDerivatives 4.0 Unported License. To view a copy of this license, visit http://creativecommons.org/licenses/by-nc-nd/4.0/

\section{Summary points}

Background

- Biosimilars of rituximab were first approved in 2017, and have the potential to improve access to biological therapies as well as the overall sustainability of cancer care.

- Real-world evidence of biosimilar uptake can help to further understand the likely impact of rituximab biosimilars for the treatment of lymphoma.

Results

- In patients with lymphoma in Germany between July 2017 and June 2019, a total of 38 different rituximab-containing therapeutic protocols were used, the most frequent being bendamustine plus rituximab and rituximab plus cyclophosphamide, doxorubicin, vincristine and prednisolone.

- A rituximab biosimilar, including Sandoz rituximab or Celltrion rituximab, was used in $55.2 \%$ of cycles overall, including $48.6 \%$ of cycles in patients with $\mathrm{FL}$ and $70.0 \%$ of cycles in patients with diffuse large B-cell lymphoma.

- Overall, in patients with non-Hodgkin lymphoma or chronic lymphocytic leukemia, a rituximab biosimilar was used in $52 \%$ at first line and $55 \%$ at second line or later.

- Switches between different rituximab medicines were frequent, including from reference rituximab to biosimilars, from biosimilars to the reference medicine and from biosimilar to biosimilar.

- Within the observation period, the proportion of rituximab biosimilar prescriptions in the total population increased from 12.0 (July 2017) to 83.0\% (June 2019).

Discussion

- Use of rituximab biosimilars is widespread in all approved indications, including extrapolated indications such as chronic lymphocytic leukemia and diffuse large B-cell lymphoma, and is increasing year-on-year.

- Real-world switching between different rituximab medicines is occurring frequently.

\section{References}

Papers of special note have been highlighted as: • of interest; $\bullet \bullet$ of considerable interest

1. Grillo-Lopez AJ, White CA, Dallaire BK et al. Rituximab: the first monoclonal antibody approved for the treatment of lymphoma. Curr. Pharm. Biotechnol. 1(1), 1-9 (2000).

-. A comprehensive summary of the development and use of rituximab across lymphoma indications.

2. ESMO Guidelines Committee. Appendix 4: Chronic lymphocytic leukaemia: eUpdate published online 27 June 2017 (www.esmo.org/Guidelines/Haematological-Malignancies). Ann.Oncol. 28(Suppl. 4), iv149-iv152 (2017).

3. EMA. Assessment report:

Rixathon (2017). www.ema.europa.eu/en/documents/assessment-report/rixathon-epar-public-assessment-report_en.pdf

4. EMA. Truxima EPAR (2016).

5. Jaeger U. Rituximab biosimilars open new horizons in immunotherapy. ESMO Open 2(3), e000234 (2017).

6. Jurczak W, Dlugosz Danecka M, Buske C. Rituximab biosimilars for lymphoma in Europe. Expert Opin. Biol. Ther. 19(10), 1045-1056 (2019).

-. A concise up-to-date overview of approved and pipeline rituximab biosimilars, including detailed overviews of clinical development programs.

7. Visser J, Feuerstein I, Stangler T, Schmiederer T, Fritsch C, Schiestl M. Physicochemical and functional comparability between the proposed biosimilar rituximab GP2013 and originator rituximab. BioDrugs 27(5), 495-507 (2013). 
8. da Silva A, Kronthaler U, Koppenburg V et al. Target-directed development and preclinical characterization of the proposed biosimilar rituximab GP2013. Leuk. Lymphoma 55(7), 1609-1617 (2014).

9. Jurczak W, Moreira I, Kanakasetty GB et al. Rituximab biosimilar and reference rituximab in patients with previously untreated advanced follicular lymphoma (ASSIST-FL): primary results from a confirmatory phase 3, double-blind, randomised, controlled study. Lancet. Haematol. 4(8), e350-e361 (2017).

10. Smolen JS, Choe JY, Prodanovic N et al. Safety, immunogenicity and efficacy after switching from reference infliximab to biosimilar SB2 compared with continuing reference infliximab and SB2 in patients with rheumatoid arthritis: results of a randomised, double-blind, phase III transition study. Ann. Rheum. Dis. 77(2), 234-240 (2018).

11. Tony HP, Kruger K, Cohen SB et al. Brief report: safety and immunogenicity of rituximab biosimilar gp 2013 after switch from reference rituximab in patients with active rheumatoid arthritis. Arthritis Care Res. (Hoboken) 71(1), 88-94 (2019).

12. Kim WS, Buske C, Ogura M et al. Efficacy, pharmacokinetics, and safety of the biosimilar CT-P10 compared with rituximab in patients with previously untreated advanced-stage follicular lymphoma: a randomised, double-blind, parallel-group, non-inferiority phase 3 trial. Lancet Haematol. 4(8), e362-e373 (2017).

13. Ogura M, Sancho JM, Cho SG et al. Efficacy, pharmacokinetics, and safety of the biosimilar CT-P10 in comparison with rituximab in patients with previously untreated low-tumour-burden follicular lymphoma: a randomised, double-blind, parallel-group, phase 3 trial. Lancet Haematol. 5(11), e543-e553 (2018).

14. Yoo DH, Suh $\mathrm{CH}$, Shim SC et al. A multicentre randomised controlled trial to compare the pharmacokinetics, efficacy and safety of CT-P10 and innovator rituximab in patients with rheumatoid arthritis. Ann. Rheum. Dis. 76(3), 566-570 (2017).

15. Yoo DH, Suh CH, Shim SC et al. Efficacy, safety and pharmacokinetics of up to two courses of the rituximab biosimilar CT-P10 versus innovator rituximab in patients with rheumatoid arthritis: results up to week 72 of a Phase I randomized controlled trial. BioDrugs 31(4), 357-367 (2017).

16. Park W, Bozic-Majstorovic L, Milakovic D et al. Comparison of biosimilar CT-P10 and innovator rituximab in patients with rheumatoid arthritis: a randomized controlled Phase 3 trial. MAbs 10(6), 934-943 (2018).

17. Park W, Suh CH, Shim SC et al. Efficacy and safety of switching from innovator rituximab to biosimilar CT-P10 compared with continued treatment with CT-P10: Results of a 56-week open-label study in patients with rheumatoid arthritis. BioDrugs 31(4), 369-377 (2017).

18. Smolen JS, Cohen SB, Tony HP et al. A randomised, double-blind trial to demonstrate bioequivalence of GP2013 and reference rituximab combined with methotrexate in patients with active rheumatoid arthritis. Ann. Rheum. Dis. 76(9), 1598-1602 (2017).

19. Jurczak W, Cohen S, Illidge TM, Silva AD, Amersdorffer J. Scientific rationale underpinning the development of biosimilar rituximab in hematological cancers and inflammatory diseases. Future Oncol. 15(36), 4223-4234 (2019).

- Uses data from the development process for a particular rituximab biosimilar to illustrate the overall principles of biosimilar development.

20. McCamish M, Woollett G. Worldwide experience with biosimilar development. MAbs 3(2), 209-217 (2011).

21. Rugo HS, Linton KM, Cervi P, Rosenberg JA, Jacobs I. A clinician's guide to biosimilars in oncology. Cancer Treat Rev. 46, 73-79 (2016).

22. Weise M, Bielsky MC, De Smet K et al. Biosimilars: what clinicians should know. Blood 120(26), 5111-5117 (2012).

-• A straightforward introduction to biosimilar concepts, from development to approval.

23. Bennett CL, Chen B, Hermanson T et al. Regulatory and clinical considerations for biosimilar oncology drugs. Lancet Oncol. 15(13), e594-e605 (2014).

24. Weise M, Kurki P, Wolff-Holz E, Bielsky MC, Schneider CK. Biosimilars: the science of extrapolation. Blood 124(22), 3191-3196 (2014).

25. Krause A, Aries PM, Berger $S$ et al. Rituximab in routine care of severe active rheumatoid arthritis: a prospective, non-interventional study in Germany. Z Rheumatol. 78(9), 881-888 (2019).

26. Horvat M, Zadnik V, Juznic Setina T et al. Diffuse large B-cell lymphoma: 10 years' real-world clinical experience with rituximab plus cyclophosphamide, doxorubicin, vincristine and prednisolone. Oncol. Lett. 15(3), 3602-3609 (2018).

27. Reiser M, Dorfel S, Hensel M et al. Rituximab in combination with chemotherapy for the treatment of chronic lymphocytic leukaemia in clinical practice. Eur. J. Haematol. 100(5), 455-464 (2018).

28. Chaoui $\mathrm{D}$, Choquet $S$, Sanhes $\mathrm{L}$ et al. Relapsed chronic lymphocytic leukemia retreated with rituximab: interim results of the PERLE study. Leuk. Lymphoma 58(6), 1366-1375 (2017).

29. Wendler J, Burmester GR, Sorensen H et al. Rituximab in patients with rheumatoid arthritis in routine practice (GERINIS): six-year results from a prospective, multicentre, non-interventional study in 2,484 patients. Arthritis Res. Ther. 16(2), R80 (2014).

30. Cziraky M, Pollock M. Real-world evidence studies (2015). www.appliedclinicaltrialsonline.com/real-world-evidence-studies

31. Christel M. "Evidence" trail elusive (2014). www.appliedclinicaltrialsonline.com/evidence-trail-elusive-0 
32. Franceschetti A, Baskett A, Baynton E, Baldock D, Karki C. Comparative analysis of biosimilar rituximab usage in treating non-Hodgkin lymphoma and rheumatoid arthritis: results from a multi-country study in Europe. Presented at ISPOR EU, Barcelona, Spain (2018). https://tools.ispor.org/research_pdfs/60/pdffiles/PHP113.pdf

33. Wiland P, Batko B, Brzosko M et al. Biosimilar switching - current state of knowledge. Reumatologia 56(4), 234-242 (2018).

34. EMA. Biosimilars in the EU (2019). www.ema.europa.eu/en/documents/leaflet/biosimilars-eu-information-guide-healthcareprofessionals_en.pdf

35. Cohen HP, Blauvelt A, Rifkin RM, Danese S, Gokhale SB, Woollett G. Switching reference medicines to biosimilars: a systematic literature review of clinical outcomes. Drugs 78(4), 463-478 (2018).

36. Wilking N, Lopez G, Meier K, Simoens S, van Harten W, Vulto A. Can we continue to afford access to cancer treatment? Eur. Oncol. Haematol. 13(2), 114-119 (2017).

- A concise summary of the growing issues with maintaining the sustainability of cancer treatment.

37. Simoens S, van Harten W, Lopez G, Vulto A, Meier K, Wilking N. What happens when the cost of cancer care becomes unsustainable? Eur. Oncol. Haematol. 13(2), 108-113 (2017).

38. Lopez G, Vulto A, Wilking N, van Harten W, Meier K, Simoens S. Potential solutions for sustaining the costs of cancer drugs. Eur. Oncol. Haematol. 13(2), 102-107 (2017).

- An elegant overview of potential solutions to issues of sustainability in cancer care, of which biosimilars represent one avenue.

39. McBride A, Balu S, Campbell K, MacDonald K, Abraham I. Subcutaneous versus intravenous rituximab in non-hodgkin lymphoma treated with R-CHOP: economic modeling for the US (ASH 2018 abstract). Blood 132, 4776 (2018).

40. Giuliani J, Bonetti A. The economic impact of biosimilars in oncology and hematology: the case of trastuzumab and rituximab. Anticancer Res. 39(7), 3971-3973 (2019). 